\title{
L’AUTOBIOGRAPHIE AU PAYS DE LA POLÉMIQUE
}

Françoise Simonet-Tenant Université de Rouen, CÉRÉdl

RESUMO

O artigo parte do percurso de Philippe Lejeune, que já no seu primeiro livro, L'autobiographie en France (1971), propunha o conceito de pacto autobiográfico, passa pela controvérsia que o opôs ao filósofo Georges Gusdorf, que o criticou por seu tratamento retórico, a visão formalista e desencarnada da escrita autobiográfica, até chegar à entrada da autoficção pelas mãos de Serge Doubrosky. Em seguida, a autora critica a polarização que se criou em torno desses dois conceitos, mostrando que ela é redutora. Apresenta três tendências que tomam o conceito de autoficção: a ortodoxa, que segue à risca o proposto por Doubrovsky; a de Jacques Lecarme, que o expande para autores do século $\mathrm{XX}$ anteriores à criação do termo; e a heterodoxa, de Vincent Colonna, que o aplica a qualquer época, sempre que o autor se coloque na obra com seu nome próprio. Além disso, após pontuar que a autoficção é sedutora, enquanto a autobiografia parece vetusta, chega à análise do que seria o espaço autobiográfico, conceito criado por Lejeune.

PALAVRAS-CHAVE: Autobiografia e autoficção. Pacto autobiográfico. Espaço autobiográfico. Philippe Lejeune. 
En France, pays volontiers batailleur, tout peut devenir objet de polémiques, y compris les genres littéraires! C'est ainsi que l'autobiographie s'est faite, ces dernières décennies, objet de passions critiques. Il est vrai que l'université française adore distinguer, catégoriser ou étiqueter et que la forêt des genres ne cesse de se peupler de nouvelles espèces: autobiographie, roman autobiographique, autoportrait, autobiographie fictive, autofiction... Et comme la biographie ne souhaitait pas demeurer en reste vis-à-vis de sa cousine " autobiographie" en pleine effervescence, elle s'est mise elle aussi à multiplier ses avatars: biographie mais aussi roman biographique et biographie romancée puis "biofiction", "fiction biographique", "biographie fictionnelle"2 et depuis peu " exofiction"3... N'y a-t-il dans cette inflation verbale que vaines ratiocinations et rodomontades de critiques, du vieux avec lequel on refait du neuf parce qu'on lui a trouvé un nouveau nom de baptême? Peut-être. Sans doute. Néanmoins, l'effort de distinction et de définition permet de mieux cerner la dynamique de la production littéraire et ses grands courants mimétiques (ou de ses effets de mode si l'on se veut plus dépréciatif). Je laisse la forêt de la biographie pour un prochain article et m'en tiens ici au maquis des écritures de soi. ${ }^{4} \mathrm{~J}$ 'avais tenté naguère pour aider mes étudiants qui n'entendaient goutte à cette forêt de mots de dresser un petit tableau (Tableau 1) aux ambitions modestes - et au succès limité, si j'en crois les confusions que j'ai continué de trouver dans les copies et les mémoires. Je le soumets tout de même au lecteur en manière de petit rappel ${ }^{5}$ : 


\begin{tabular}{|c|c|c|c|c|c|c|}
\hline & $\begin{array}{c}\text { Contrat avec } \\
\text { lecteur }\end{array}$ & $\begin{array}{c}\text { Identité } \\
\text { onomastique } \\
\text { (auteur/narrateur/ } \\
\text { Héros) }\end{array}$ & $\begin{array}{c}\text { Teneur du } \\
\text { récit : } \\
\text { vraisemblable }\end{array}$ & $\begin{array}{l}\text { Teneur du } \\
\text { récit : } \\
\text { vérifiable }\end{array}$ & Exemple & $\begin{array}{l}\quad \text { Bilan: } \\
\text { Ce que laisse } \\
\text { entendre } \\
\text { l'auteur au } \\
\text { lecteur, } \\
\text { explicitement } \\
\text { ou } \\
\text { tacitement } \\
\end{array}$ \\
\hline $\begin{array}{l}\text { Autobiographie } \\
\text { (au sens strict tel } \\
\text { qu'il a été défini } \\
\text { par Lejeune) }\end{array}$ & $\begin{array}{c}\text { Pacte } \\
\text { Autobiographique }\end{array}$ & Identité nécessaire & Oui & Oui & $\begin{array}{c}\text { Les } \\
\text { Confessions } \\
\text { Rousseau }\end{array}$ & “C’est moi" \\
\hline \begin{tabular}{l}
\multicolumn{1}{c}{ Autoportrait } \\
(ce qui \\
distinguerait \\
l'autoportrait de \\
l'autobiographie : \\
son organisation \\
thématique alors \\
que \\
l'autobiographie \\
privilégierait une \\
organisation \\
temporelle)
\end{tabular} & $\begin{array}{c}\text { Pacte } \\
\text { Autobiographique }\end{array}$ & Identité nécessaire & Oui & Oui & $\begin{array}{c}\text { Un petit } \\
\text { bourgeois } \\
\\
\text { François } \\
\text { Nourrissier }\end{array}$ & “C’est moi” \\
\hline $\begin{array}{c}\text { Roman } \\
\text { autobiographique }\end{array}$ & $\begin{array}{l}\text { Contrat } \\
\text { ambigu }\end{array}$ & $\begin{array}{l}\text { Identité facultative } \\
\text { (souvent partielle) }\end{array}$ & Oui & $\begin{array}{c}\text { Non } \\
\text { nécessairement }\end{array}$ & $\begin{array}{l}\text { L'Enfant } \\
\text { Jules Vallès }\end{array}$ & $\begin{array}{l}\text { "C'est peut- } \\
\text { être moi" }\end{array}$ \\
\hline Autofiction & $\begin{array}{c}\text { Pacte } \\
\text { oxymorique }\end{array}$ & $\begin{array}{c}\text { Identité nécessaire } \\
\text { (dans la } \\
\text { perspective de } \\
\text { La définition de } \\
\text { Doubrovsky) }\end{array}$ & $\begin{array}{c}\text { Non } \\
\text { nécessairement }\end{array}$ & $\begin{array}{c}\text { Non } \\
\text { nécessairement }\end{array}$ & $\begin{array}{c}\text { Fils } \\
\text { Doubrovsky }\end{array}$ & $\begin{array}{c}\text { “C'est moi et } \\
\text { ce n'est pas } \\
\text { moi" }\end{array}$ \\
\hline $\begin{array}{c}\text { Autobiographie } \\
\text { fictive / } \\
\text { Mémoires fictifs }\end{array}$ & $\begin{array}{c}\text { Pacte } \\
\text { romanesque }\end{array}$ & Disjonction & $\begin{array}{c}\text { Non } \\
\text { nécessairement }\end{array}$ & Non & $\begin{array}{l}\text { La Vie de } \\
\text { Marianne } \\
\text { Marivaux }\end{array}$ & $\begin{array}{c}\text { “Ce n'est pas } \\
\text { moi mais je } \\
\text { fais comme } \\
\text { si c'était } \\
\text { moi" }\end{array}$ \\
\hline
\end{tabular}

Tableau 1 
Inévitablement schématique, ce tableau tente de clarifier ce paysage brouillé. Nous allons revenir sur le trio "autobiographie-roman autobiographique-autofiction" qui a fait couler beaucoup d'encre pour tenter de cerner l'histoire critique voire polémique des rapports entre ces trois genres.

\section{Le pacte autobiographique, controverses et réactions}

\section{La controverse entre Lejeune et Gusdorf}

Remontons un demi-siècle en arrière. En 1968, une encyclopédie thématique demande à un jeune universitaire, Philippe Lejeune, d'écrire un article sur un genre littéraire. L'autobiographie n'est pas prévue dans le plan de l'encyclopédie: il la fait rajouter et travaille à un article qui ne sera finalement pas publié, l'encyclopédie ayant changé de formule. La rédaction de l'article lui a cependant permis de prendre conscience d'un vide: il n'existe aucun livre sur l'autobiographie française. Pendant dix-sept ans (1969-1986), il va alors se consacrer à l'étude de l'autobiographie, cycle qui s'ouvre par la publication d'un petit ouvrage à la fois, historique, théorique et anthologique, L'autobiographie en France (1998). L'expression "pacte autobiographique" figure déjà dans L'autobiographie en France. Vingt-cinq ans plus tard, Philippe Lejeune commente sa trouvaille lexicale:

À quoi renvoie "pacte"? Sans doute à une idée juridique de "contrat", mais évidemment on pense aussi à une alliance mystique ou surnaturelle $[\ldots]$ C'est un peu exagéré, mais cet excès frappe l'imagination et a assuré le succès de la formule. Je ne suis pas un théoricien révolutionnaire, mais plutôt un publicitaire qui a eu une bonne idée (LEJEUNE, 2005, p. 15).

L'expression est élevée un peu plus tard à la dignité de titre. Le pacte autobiographique est publié en 1975, conquiert progressivement son public, est traduit en de multiples langues qui donnent à son auteur une reconnaissance et une visibilité internationales.

Entre 1975 et 1986, la définition donnée par Philippe Lejeune de l'autobiographie s'est légèrement infléchie. Dans Le pacte autobiographique, la définition bien connue de l'autobiographie est la suivante: "Récit rétrospectif en prose qu'une personne réelle fait de sa propre existence, lorsqu'elle met l'accent sur sa vie individuelle, en particulier sur l'histoire de sa personnalité" (LEJEUNE, 1996, p. 14).

Dans "Le pacte autobiographique (bis)" (LEJEUNE, 1986, p. 13- 
35), Lejeune n'hésite pas à faire son autocritique: il apporte des nuances et revient sur des positions trop normatives. Dans Le pacte, il écrivait: “Une identité est, ou n'est pas. Il n'y a pas de degré possible, et tout doute entraîne une conclusion négative" (LEJEUNE, 1996, p. 15). À la relecture, il se dit frappé par l'intransigeance de son attitude et il s'aperçoit que dans l'analyse même des textes et à travers la notion d' "espace autobiographique" qu'il inaugurait avec une étude d'œuvres gidiennes, il admet pourtant l'existence d'ambiguïtés et de degrés.

En outre, il revient dans Moi aussi sur le rapport entre autobiographie et écriture poétique:

Le paradoxe de l'autobiographie littéraire, son essentiel double jeu, est de prétendre être à la fois un discours véridique et une œuvre d'art. C'est pour avoir médité sur cette quadrature du cercle et tenté de réaliser cet équilibre que Michel Leiris est si exemplaire. Aussi, plutôt que de proposer une définition qui, sèchement, exclut les vers, aurais-je dû désigner comme centre du système actuel cette tension entre la transparence référentielle et la recherche esthétique (LEJEUNE, 1986, p. 26).

Le grand mérite de Lejeune est d'avoir permis la reconnaissance d'un genre non fictionnel et d'une écriture véridictionnelle dans le champ littéraire et d'avoir montré que l'art ne fraye pas avec la seule fiction. Son apport consiste aussi en la définition de l'autobiographie comme genre performatif et référentiel et en la proposition d'une conception contractuelle du texte autobiographique manifestée par 1' "identité de nom entre l'auteur (tel qu'il figure, par son nom, sur la couverture), le narrateur du récit et le personnage dont on parle" (LEJEUNE, 1996, p. 23-24): l'autobiographe, en son nom propre et en son propre nom, promet au lecteur que ce qu'il va lui dire est vrai, ou du moins est ce qu'il croit vrai. Enfin, la théorie de Lejeune, contrairement à ce que certains ont pu dire, est souple et apparaît plutôt comme un work in progress que comme un système figé.

Dès les premiers travaux de Lejeune a éclaté une controverse. Son approche suscite les critiques de Georges Gusdorf. C'est un philosophe qui, en France, a exploré le premier l'histoire de l'autobiographie: en témoignent son ouvrage La découverte de soi (1948) et un article fondateur consacré aux Conditions et limites de l'autobiographie (1956). Gusdorf reproche à Lejeune un traitement trop rhétorique et une vision formaliste et désincarnée de l'écriture autobiographique. ${ }^{5} \mathrm{Il}$ critique la revendication 
par Lejeune du caractère moderne de l'autobiographie qui voit dans le $\mathrm{XVIII}^{\mathrm{e}}$ siècle, et plus particulièrement dans Rousseau, une rupture constitutive. C'est une première controverse dont nul ne sort véritablement victorieux. Néanmoins il est évident que les travaux de Lejeune s'imposent dans la sphère universitaire, au-delà des strictes frontières francophones et que ses talents de vulgarisateur lui permettent de trouver sa place dans le champ médiatique.

\section{Le défi relevé par Doubrovsky}

La deuxième réaction notable aux travaux de Lejeune est fort différente: elle n'opère pas tant sur le mode de la controverse que sur celui de l'émulation créative. Serge Doubrovsky, universitaire connu pour ses travaux critiques sur Sartre et Corneille, était plongé dans la genèse de son roman Fils quand il lut Le pacte autobiographique. Philippe Lejeune y suggérait qu'il n'y aurait pas d'exemple de texte où coexisteraient l'identité onomastique de l'auteur, du narrateur et du personnage et un pacte romanesque. Doubrovsky se prend au jeu: il va fournir par le livre même qu'il est en train d'écrire un exemple au poéticien. L'autofiction était née, dont l'acte de baptême figure sur la quatrième de couverture de Fils (1977):

Autobiographie? Non, c'est un privilège réservé aux importants de ce monde, au soir de leur vie, et dans un beau style. Fiction, d'événements et de faits strictement réels; si l'on veut, autofiction, d'avoir confié le langage d'une aventure à l'aventure du langage, hors sagesse et hors syntaxe du roman, traditionnel ou nouveau.

L'on peut s'étonner que la définition donnée par Doubrovsky de l'autobiographie - "privilège réservé aux importants de ce monde, au soir de leur vie" - qui lui sert de repoussoir à celle d'autofiction corresponde finalement mieux au genre des mémoires qu'à celui de l'autobiographie: en effet, contrairement aux autres récits de soi, le mémorialiste se distingue par une autorité, qu'elle soit de nature sociale, politique ou militaire, qui fait de lui un "important de ce monde" et qui veut, en tant qu'acteur de l'Histoire, conserver par écrit un mémorable destiné à être examiné par la postérité. Cette réserve énoncée, on observe que cette définition fondatrice de l'autofiction insiste sur la littérarité du texte et sa capacité d'innovation. Cette définition a été suivie d'autres, en particulier dans l'article "Autobiographie / vérité / psychanalyse" (DOUBROVSKY, 
1988) où Doubrovsky montre que l'autofictionnaire met en pratique dans l'écriture les procédés psychanalytiques de déplacement et de condensation. La dernière mise au point, finalement plus inconsistante, de Doubrovsky date de 2011 dans un numéro de La Nouvelle Revue Française, intitulée "Je et moi": "je donnerais ultimement cette définition de l'autofiction: récit dont la matière est entièrement autobiographique, la manière entièrement fictionnelle. [...] l'autofiction est finalement la forme contemporaine de l'autobiographie" (DOUBROVSKY, 2011, p. 23-25).

Le "pacte autobiographique" et l' "autofiction" sont deux expressions qui ont fait mouche et qui ont suscité, sans que leurs créateurs l'aient voulu, l'organisation durable du champ des écritures de soi en deux pôles, le pôle autofiction et le pôle autobiographie, bipartition réductrice et médiocrement opératoire.

\section{Emballement critique et dilution médiatique}

1977-2011: se passent près de trente-cinq années pendant lesquelles l'autofiction va faire couler beaucoup d'encre et susciter nombre de polémiques qui débordent les milieux universitaires pour envahir certains supports médiatiques. Pour les détracteurs de l'autofiction, celle-ci n'est qu'une étiquette pour rajeunir un vieux genre grâce à laquelle l'écrivain fait passer en fraude la vie sous l'étiquette du roman. Quant au camp des autofictionnaires, ils ne parlent point d'une seule voix et trois tendances apparaissent progressivement: ${ }^{6}$

- Les orthodoxes attachés au sens initial donné par Doubrovsky;

- Ceux qui vont assigner au terme un domaine plus large. C'est le cas de Jacques Lecarme: celui-ci fait observer que, quelques décennies avant Fils et sans user du terme "autofiction" Pierre Loti (Le roman d'un enfant), Colette (La naissance du jour), Céline (la trilogie allemande), Léautaud (Le petit ami) appliquaient déjà la formule proposée par Doubrovsky (voir LECARME, 2004, p. 13-23). On peut également rattacher à ce mouvement d'élargissement l'acception d' "autofiction" à laquelle aboutit Philippe Vilain, rendant facultatif le trait définitoire de l'identité onomastique (VILAIN, 2009, p. 74); ${ }^{7}$

- Les hétérodoxes qui, tel Vincent Colonna, s'emparent du mot mais en transforment le sens. En effet, à ses yeux, l'autofiction “consiste à s'inventer des aventures que l'on s'attribuera, à 
donner son nom d'écrivain à un personnage introduit dans des situations imaginaires" (COLONNA, 1989, p. 3). Dans ce dernier cas, l'homonymat devient le tremplin d'une mise en scène autofabulatrice qui projette l'auteur dans un univers fictif, c'est-à-dire un état non avéré du monde. C'est ainsi que Colonna donne comme exemple paradigmatique de l'autofiction La divine comédie, voyage imaginaire dont le narrateur-personnage est Dante.

La fortune critique du terme lancé par Doubrovsky n'a d'égal que son détournement jusqu'à sa dilution médiatique. Un des raisons possibles du succès du terme est sans doute la présence dans ce néologisme composé de la consolante "fiction" qui, depuis Aristote, se confond peu ou prou avec la littérature. Alors que l'autobiographie ramène dangereusement du côté de la vie et de l'écriture, voire des écritures ordinaires, l'autofiction rassure: on n'a pas quitté le champ de la littérature.

\section{Conflits de voisinage au pays des écritures de soi}

\section{L'autobiographie: une étiquette qui passe mal}

L'autobiographie reste une étiquette qui passe mal auprès des auteurs. De Sarraute à Modiano en passant par Jacques Roubaud, on récuse ce terme à l'odeur de soufre. À Viviane Forester, Nathalie Sarraute affirme à propos d'Enfance: “Il ne s'agit pas d'une autobiographie. Ce n'est pas un rapport sur ma vie. Le souvenir lorsqu'il n'est pas repris dans le travail est tellement grossier" (FORRESTER, 1983, p. 19). Dominique Viart et Bruno Vercier, auteurs d'un ouvrage récent consacré à La littérature française au présent, ne manquent pas d'observer que, si l'autobiographie prolifère, le mot lui-même est suspect. "Les écrivains qui la pratiquent préfèrent en inventer d'autres" (VIART; VERCIER, 2005, p. 27), et les auteurs de dresser une liste non exhaustive: l'automythobiographie de Claude Louis-Combet, l'autobiogre d'Hubert Lucot, les otobiographies de Jacques Derrida, la prose de mémoire de Jacques Roubaud, l'ego-littérature de Philippe Forest. Rares sont les auteurs contemporains qui ont revendiqué ou revendiquent haut et fort un engagement autobiographique: l'on peut citer dans cette catégorie d'autobiographes acharnés et le revendiquant Jacques Borel, Charles Juliet et Annie Ernaux. 
Du côté de la critique et de l'institution universitaire, le terme "autobiographie" même s'il a fini par se faire une place jusque dans les programmes universitaires et les sujets de concours suscite encore bien des réticences, et les nostalgiques des années structuralistes et de "la mort de l'auteur" n'en reviennent toujours pas de l'importance croissante pris par les écritures de soi dans le champ critique. Il arrive à Philippe Lejeune d'être brocardé, et ce pour deux raisons essentielles. Comment peut-il parler de discours dans un esprit de vérité à une époque où la psychanalyse nous a enseigné les chausse-trappes du sujet et ses illusions identitaires? Et beaucoup de citer jusqu'à plus soif Lacan et de se gargariser avec la formule désormais bien connue: "Le moi, dès l'origine serait pris dans une ligne de fiction" (LACAN, 1966, p. 94). Second reproche fait à Lejeune: comment peut-il accorder de l'intérêt aux écritures autobiographiques ordinaires au risque de mettre en péril la littérature?

\section{Autofiction et autolégitimation}

Tandis que l'autobiographie est considérée avec méfiance, toujours suspectée de crime lèse- littérature, l'autofiction s'érige en place forte. Parce qu'on n'est jamais mieux servi que par soi-même, les autofictionnaires décidés à s'autolégitimer, participant aux colloques et s'épanchant dans des ouvrages et articles critiques, multiplient les commentaires et les discours d'escorte. Il faut dire que d'emblée Doubrovsky avait donné le ton, à la fois critique, universitaire, écrivain, théoricien. Ses épigones suivront le même chemin mélangeant les genres avec maestria, se voulant à la fois romanciers et critiques. Un exemple très caractéristique de ce brouillage est celui de Philippe Vilain, auteur d'une œuvre romanesque et de plusieurs essais, dont Défense de Narcisse (2005) et L'autofiction en théorie (2009). Habitué des colloques, il n'hésite pas à faire de la critique génétique sur ses propres œuvres, démarche aussi ambivalente qu'ambiguë. Ainsi lors de la journée d'étude "Genèse et autofiction" qui s'est tenue à l'École Normale Supérieure le 4 juin 2005, Philippe Vilain, invité, se mit à analyser ses propres brouillons. Relire ses brouillons, a-t-il constaté, c'est un peu descendre à la cave. On croit savoir ce qu'on y a rangé et ce n'est pas le cas: l'idée qu'on se fait de ses propres manuscrits ne correspond pas à la réalité. Il a tenté d'observer la "réinvention du référentiel" à partir de ses propres traces et s'est livré à deux analyses d'extraits de L'étreinte et de La derniere annee. Du je des manuscrits au je assumé publié, il est allé, selon lui, du ressassement du référentiel à 
l'autofiction qui est un moyen de refigurer son histoire personnelle en la déplaçant et dans laquelle le moi peut se dissimuler à sa guise.

\section{Le retour du roman autobiographique?}

L' "autofiction" passant aux yeux de certains pour un terme à la mode, apparaissant aux yeux d'autres comme une notion trop vague, l'on voit revenir sur le marché des genres littéraires l'étiquette désuète de "roman autobiographique" qui avait été mise par Jacques Lecarme au magasin des accessoires (voir LECARME; LECARME-TABONE, 1997, p. 24). Néanmoins, coup sur coup, deux théoriciens, Yves Baudelle et Philippe Gasparini, vont appeler à la rescousse la formule du roman autobiographique, justifiant leur démarche par des arguments qui méritent d'être pris en compte.

C'est également au roman autobiographique que se réfère Philippe Lejeune lorsqu'il explique l'origine de son grand chantier sur l'autobiographie: "Toute mon analyse était partie d'une évidence: comment distinguer l'autobiographie du roman autobiographique?" (LEJEUNE, 1986, p. 25). Rappelons que dans l'autobiographie, l'auteur conclut avec le lecteur un pacte qui revient à dire au lecteur: "C'est moi dont je parle". Dans le roman autobiographique, l'auteur remplit avec le lecteur un constat ambigu: 'C'est peut-être moi dont il s'agit mais ce n'est pas certain." Le critère essentiel de distinction de l'autobiographie, l'identité onomastique (auteur/narrateur/personnage), n'est pas vérifié dans le roman autobiographique. C'est intentionnellement que le romancier autobiographe joue avec le lecteur à cache-cache, ne souhaitant pas, pour diverses raisons, assumer l'identité auteur-narrateur-personnage et aiguisant par là même le voyeurisme du lecteur transformé en limier. Il appartient à Philippe Gasparini, au temps de l'autofiction triomphante, d'avoir remis sur le devant de la scène la notion de roman autobiographique dans Est-il je? - roman autobiographique et autofiction. Si une modernité oublieuse porte aux nues une autofiction à la génération prétendument spontanée, l'essai de Gasparini rappelle utilement que les rapports entre roman et autobiographie ont été posés bien avant l'ère de l'autofiction. Il établit que roman autobiographique et autofiction seraient des sous-genres connexes:

Le roman autobiographique s'inscrit dans la catégorie du possible [...], du vraisemblable naturel. Il doit impérativement convaincre le lecteur que tout a pu se passer logiquement de cette manière. Faute de 
quoi il bascule dans un autre genre qui, lui, mélange vraisemblable et invraisemblable, l'autofiction (GASPARINI, 2004, p. 29).

Dans la mesure où l'autofiction est une catégorie aussi floue que vampirique, cette distinction peut laisser le lecteur sceptique. Peut-être la tentative par Yves Baudelle de discrimination d'avec l'autofiction du roman autobiographique réhabilité est-elle plus convaincante. Reprenant la pierre de touche de distinction de ces sous-genres connexes, il observe avec pertinence que "dans l'autofiction, tout peut être faux, sauf le nom principal. Dans le roman autobiographique, au contraire, tout peut être vrai, sauf les noms. La loi d'airain du roman autobiographique, c'est de changer les noms (mais s'il ne change que les noms, il n'est plus qu'un roman à clés)" (BAUDELLE, 2003, p. 21).

Le roman autobiographique, catégorie certes surannée, n'est sans doute ni moins fragile, ni moins opératoire que la catégorie cousine, aux couleurs de modernité, qu'est l'autofiction. Néanmoins les querelles de voisinage générique et terminologique et la résurrection d'un genre désuet sont-elles la bonne manière pour sortir des polémiques? Si l'on peut s'accorder sur le fait que "la forme canonique du récit rétrospectif en prose, avec son pacte de sincérité, son ordonnance chronologique, son ambition à ressaisir la totalité d'une vie, a cédé du terrain devant des avatars plus modernes et plus chatoyants" (MONTÉMONT, 2013, p. 55), est-il nécessaire de multiplier les étiquettes pour rendre compte des métamorphoses du champ autobiographique? Ne serait-il pas préférable d'exploiter plus largement une notion mise à jour par Philippe Lejeune dès Le pacte autobiographique, la notion d' "espace autobiographique" qui permet de considérer l'intervalle problématique voire indécidable entre roman et autobiographie?

\section{Et si on reparlait d" "espace autobiographique"? Définition de la notion}

A priori l'on pourrait penser que l'espace autobiographique désigne les textes non ouvertement assumés par l'auteur comme textes autobiographiques, mais qui empruntent des matériaux aspirés à sa vie réelle. Ce serait cependant réduire la notion d'espace autobiographique à l'idée floue et approximative d'inspiration autobiographique alors qu'elle conduit bien au-delà de ce rétrécissement anecdotique. 
Lorsque Philippe Lejeune fait apparaître l'expression dans Le pacte autobiographique, c'est dans un sens plus précis et opératoire. La notion s'applique particulièrement aux auteurs qui "ont choisi de laisser leur autobiographie incomplète, fragmentée, trouée et ouverte "et qui conduisent ainsi "leurs lecteurs à lire dans le registre autobiographique tout le reste de leur production narrative" (LEJEUNE, 1996, p. 43). L'exemple spectaculaire choisi par Philippe Lejeune pour illustrer cette notion est celui de l'œuvre gidienne, architecture subtile où entrent en résonance textes fictifs, essayistes et autobiographiques. L'image de l'être de dialogue, voire de contradictions qu'était Gide, ne peut s'enfermer dans une œuvre monolithique, de là un espace polyphonique où peut se dire l'instabilité de l'image de soi. Les récits gidiens prennent un autre relief grâce à la présence dans l'ensemble de l'œuvre d'un texte explicitement autobiographique et assumé comme tel, Si le grain ne meurt... Si plusieurs récits de Gide sont saturés d'éléments empruntés au vécu (notamment L'immoraliste et La porte étroite), ils ne peuvent cependant être assimilés à de simples transpositions biographiques; ils participent néanmoins de "l'espace autobiographique" constitué par l'essentiel de l'œuvre et peuvent être lus comme des fantasmes significatifs des tourments d'un individu.

Prenons pour exemple La porte étroite. Le récit - fractionné en huit chapitres, quelques pages du journal d'Alissa et un épilogue - est l'histoire de l'amour de Jérôme et de sa cousine, Alissa Bucolin, qui appartiennent tous deux à la bourgeoisie protestante. La tendresse qui lie les deux cousins devient un amour profond dont Jérôme attend un bonheur humain alors qu'Alissa repousse celui qu'elle aime, tendue vers un absolu spirituel et persuadée que Dieu les a "réservés pour quelque chose de meilleur" (GIDE, 1958, p. 578). Cette ascèse implacable la conduit à une mort solitaire dont rendent compte les pages de son journal qu'elle a légué à Jérôme.

André Gide, dans les textes postérieurs à l'écriture de La porte étroite, s'est élevé avec vivacité contre l'identification trop facile qui pouvait être faite de son épouse Madeleine à Alissa. Sans doute cette objection a-t-elle sa raison d'être car, comme l'a analysé Philippe Lejeune, on ne saurait ignorer la subtilité et l'ambiguïté du récit gidien.

Malaise $[\ldots]$ des lecteurs actuels, devant l'évanescence de l'œuvre: cette espèce de contradiction ou de perplexité au niveau de la lecture est justement l'effet de "l'espace autobiographique" gidien, qui exclut 
à la fois la seule lecture romanesque des textes et leur pure réduction biographique (LEJEUNE, 1996, p. 187).

Le roman, autobiographie du possible: sans doute est-ce la définition qui pouvait convenir à Gide, laissant croître jusqu'à l'extrême dans les personnages de ses récits un trait ou un comportement repérés chez lui ou autrui. C'est d'autant plus vrai dans La porte étroite qui constitue finalement la reprise fictionnelle du problème conjugal qui s'est posé à Gide: à cet égard, le récit constitue un drame à la fois dépassé (puisque les deux cousins, André et Madeleine, ont fini par se marier) et irrésolu (puisque leur mariage n'a pas été consommé), un “drame qui n'a pas achevé de se jouer" (GIDE, 1958, p. 430), comme Gide le reconnaîtra au moment de l'écriture de Si le grain ne meurt. C'est donc à une résolution possible du conflit de l'amour idéal et de la possession charnelle que s'essaie Gide: il le résout tragiquement par la mort de l'héroïne, choix fictionnel radical qui fut assez souvent le sien (voir L'immoraliste). Cette solution romanesque s'offre à l'écrivain, retenu d'être autobiographe par l'impossibilité où il se trouve de résoudre, autrement que par l'échappée fictionnelle, ce que la vie n'a pas encore conclu.

\section{Son intérêt critique}

La notion d'espace autobiographique s'avère efficace pour appréhender maintes œuvres autobiographiques publiées après 1945. La vulgarisation de la psychanalyse a changé au cours du $\mathrm{xx}^{\mathrm{e}}$ siècle le rapport qu'on pouvait avoir à son for intérieur: la croyance en la transparence d'un sujet immobilisé est révolue; l'on est conscient qu'un projet de dévoilement total de soi tient pour une large part du mirage. De là, la raréfaction, ces dernières décennies, des grandes entreprises autobiographiques totalisatrices, sur le modèle d'un Rousseau ou d'un Chateaubriand; de là, le règne des autobiographies lacunaires et parcellaires voire hybridées avec des fictions narratives ou de la prose essayiste. On a tout à gagner à recourir à la notion d'espace autobiographique pour analyser ces formes contemporaines. L'espace autobiographique suggère l'idée qu'il y a du jeu dans l'autobiographie au sens "d'espace ménagé pour la course d'un objet": le je narrant et le je narré sont pris dans un mouvement perpétuel et la notion d'espace autobiographique permet d'échapper au je monolithique, d'intégrer l'idée d'une instabilité identitaire et l'image fragmentaire d'un moi saisi sous différentes perspectives. Avec l'espace 
autobiographique on n'est plus du côté du soi statufié, du soi fixé comme d'outre-tombe. On a quitté le monumental pour le parcellaire.

L'espace autobiographique permet en outre de rendre compte des écrivains qui cultivent non seulement la parcellisation mais également l'ambiguïté. C'est par exemple le cas de Patrick Modiano qui, depuis 1968, a publié plus de vingt-cinq récits et romans, véritable puzzle de personnages, lieux et situations récurrents, baigné par une esthétique qui mêle hyperréalisme et onirisme et par l'ombre de l'Occupation allemande en France, période dont Modiano affirme être issu. Quand paraît Un pedigree en 2005, récit factuel, lapidaire et ascétique aux allures de curriculum vitae, les lecteurs retrouvent des faits et des lambeaux de vie dont ils ont déjà vu la transposition dans des romans antérieurs, lesquels prennent du même coup, ainsi éclairés, un autre relief. Néanmoins dans un entretien donné à Lire (mars 2010), Modiano répond à propos d'Un pedigree:

On pourrait classer ce livre du côté des autobiographies - c'est d'ailleurs ce qu'on a fait - mais j'ai toujours eu l'impression que ce livre se rattachait aux romans. En fait, la perspective de l'autobiographie m'a toujours perturbé. Dans Un pedigree, je ne racontais pas une vie, la mienne. Je parlais de choses qui m'avaient été imposées. [...] Je parlais de choses qui m'avaient fait souffrir mais qui m'étaient étrangères, qui ne m'étaient pas intimes. Bien sûr, il s'agissait de mes parents. Mais ces choses m'avaient été imposées par eux et étaient presque comme des corps étrangers. J'ai écrit ce livre pour me débarrasser de ces éléments étrangers, pas pour raconter ma vie (MONDIANO in BUSNEL, 2010).

Réponse pour le moins ambiguë, encore brouillée par une assertion qui vient un peu plus loin dans l'entretien: "Mes livres sont faits de bric et de broc autobiographique". L'œuvre modianesque travaillée par un mouvement contradictoire d'attirance et de résistance vis-à-vis de l'écriture autobiographique finit bien par construire un espace autobiographique, fait de traits biographiques et de fables (voir "Modiano", CAMPOS dans SIMONET-TENANT, 2017, p. 558-559).

Autobiographie, autofiction, roman autobiographique: au-delà des polémiques et des querelles de mots se joue sans doute ici une réflexion sur l'idée qu'on se fait de la littérature, et sur les frontières - infranchissables ou non - entre fiction et non-fiction. Autobiographie, autofiction, roman autobiographique: est-il entre ces genres simplement une question de degré ou de nature? Pour sortir de l'impasse de cette question 
dichotomique s'offre la notion d'espace autobiographique: en accueillant en son sein l'écriture véridictionnelle et l'écriture fictive, l'espace autobiographique permet une cohabitation possible des deux régimes avec l'éventualité de phénomènes d'hybridation de l'un et de l'autre et de diffraction de l'un sur l'autre.

\section{THE AUTOBIOGRAPHY IN THE COUNTRY OF POLEMIC}

\section{ABSTRACT}

This article has Philippe Lejeune's pathway as its point of departure, for having introduced the concept of the autobiographical pact in L'autobiographie en France (1971), his first book. It then visits his controversy with philosopher Georges Gusdorf, who criticized him for his rhetorical approach, his formalist and disembodied perspective of autobiographic writing. Finally, it renders the rise of autofiction by Serge Doubrovsky. Following, the article criticizes the polarization between both concepts, by demonstrating how reductionist it can be. The concept of autofiction is then explored in three main trends: the orthodox, which strictly follows Doubrovsky's proposal; Jacques Lecarme's, which expands it to encompass $20^{\text {th }}$-century authors previous to the term's coinage; and Vicent Colonna's heterodox trend, which applies it to any historical period whenever an author nominally inserts himself into his work. Besides, after comparing autofiction's seductiveness to autobiography's apparent ancientness, the text analyzes the concept of the autobiographical space, created by Lejeune.

KEYWORDS: Autobiography. Autofiction. Autobiographical Pact. Autobiographical Space. Philippe Lejeune 


\section{NOTAS}

${ }^{1}$ On doit ce néologisme à Alain Buisine. La biofiction est un texte littéraire dont le cadre narratif épouse celui de la biographie.

${ }^{2}$ PUECH (2013) distingue les fictions biographiques qu'il définit comme des "variations romanesques sur les biographies d'auteurs réels" des "biographies fictionnelles" où il voit des "pastiches de biographies (pastiches tant formels que thématiques) créant des auteurs imaginaire". On pourrait étendre l'expression de "fictions biographiques" aux variations romanesques sur les biographies de personnes réelles quand bien même elles seraient des mathématiciens (DESERABLE, 2015), des artistes (ECHENOZ, 2006) ou des individus plus obscurs sur le berceau desquels les fées de la Renommée ne se sont pas penchées (GOULET, 2011). RABATE (2006) voit dans la fiction biographique un entre-deux générique entre fiction et biographie. GEFEN (2015) fait du modèle biographique un mode de renouvellement de l'écriture romanesque dans le sillage de SCHWOB (1896) à qui l'on doit "le tournant fictionnel de la biographie".

${ }^{3}$ On devrait ce terme formé en opposition à "autofiction" à l'écrivain Philippe Vasset (dans ROUSSEL, 2013). Les exofictions seraient selon Vasset des récits construits "à partir d'énigmes que nous présente le réel."

${ }^{4}$ Voir SIMONET-TENANT, 2017, p. 290: "Écritures du moi" et "écritures de soi" semblent a priori des expressions équivalentes. Encore un examen un peu plus attentif de leur utilisation dans le champ critique permet-il de mesurer qu'elles sont porteuses de connotations sensiblement différentes. C'est à GUSDORF (1991) que l'on peut attribuer l'expression "écritures du moi". Hostile à toute approche formaliste de l'autobiographie, il s'inscrit dans la lignée du philosophe Georg Misch (1878-1965), auteur d'une somme en quatre volumes sur l'histoire de l'autobiographie de l'Antiquité au XIX siècle. Il y a chez Gusdorf la volonté de poursuivre l'étude de Misch jusqu'aux temps contemporains et une conception très large des écritures du moi vues comme "des figures fugitives sur le sable et l'eau de la vie", qui "exposent des attestations de la présence humaine sur la terre des vivants, indissociables des cycles et rythmes de la conscience individuelle et de la conscience communautaire qui se prononcent à travers elle" (GUSDORF, 1991). Des deux expressions “écritures du moi” et "écritures de soi”, c'est la seconde qui eut la plus grande fortune critique. Sans doute la doit-on à FOUCAULT (1983). Quasiment considérée par LEJEUNE (2005) comme un euphémisme pour contourner des "mots malsonnants" tels qu'autobiographie ou témoignage - médiocrement appréciés dans les milieux littéraires car soupçonnés de délit de lèselittérarité -, elle permet également de faire l'économie du moi substantivé auquel 
s'attache, depuis le XVII siècle et Pascal, une connotation dévalorisante. "Écritures de soi" introduit une distanciation bienséante, éloigne les menaces de l'égocentrisme et de l'égotisme, donne un gage de respectabilité littéraire - conforme en un sens à une certaine représentation de la littérature qui voudrait qu'elle fût le "passage du Ich au Er, du Je au Il" (BLANCHOT, 1949) - et sert de locution générique pour englober une vaste catégorie d'écrits."

${ }^{5}$ Voir Revue d'Histoire Littéraire de la France, n. 6, nov.-déc. 1975.

${ }^{6}$ Voir “Autofiction” (JOUANNY dans SIMONET-TENANT, 2017, p. 94-97).

${ }^{7}$ Vilain définit ainsi l'autofiction: "Fiction homonymique ou anominale qu'un individu fait de sa vie ou d'une partie de celle-ci."

\section{REFERÊNCIAS}

BAUDELLE, Yves. Du roman autobiographique: problèmes de la transposition fictionnelle. Protée, v, 31, n. 1, p. 7-26, Printemps 2003.

BLANCHOT, Maurice. La part du feu. Paris: Gallimard, 1949.

BUSNEL, François. Modiano: «Mon Paris n'est pas un Paris de nostalgie mais un Paris rêvé». Lire, mar. 2010. Disponível em: <https://www.lexpress.fr/culture/livre/modiano-mon-paris-n-est-pas-un-paris-de-nostalgie-mais-un-paris-reve_852746.html>.Acesso em: 10 nov. 2017.

CAMPOS, Laura. Mondiano. In: SIMONET-TENANT, Françoise (Org.). Dictionnaire de l'autobiographie; écritures de soi de langue française. Paris: Honoré Champion, 2017, p. 558-559.

COLONNA, Vincent. L'Autofiction: essai sur la fictionnalisation de soi en littérature. Tese (Doutorado em Linguística) - Ecole des Hautes Etudes en Sciences Sociales, Paris, 1989.

DÉSÉRABLE, François-Henri. Évariste. Paris: Gallimard, 2015.

DOUBROVSKY, Serge. Fils. Paris: Galilée, 1977. . Autobiographie / vérité / psychanalyse. In: . Autobiographiques: de Corneille à Sartre. Paris: PUF, 1988.

. C'est fini. La Nouvelle Revue Française, n. 598, p. 23-25, oct. 2011.

ECHENOZ, Jean. Ravel. Paris: Minuit, 2006.

FORRESTER, Viviane. Portrait de Nathalie. Le Magazine Littéraire, n. 196, p. 18-21, jun. 1983. 
FOUCAULT, Michel. L'écriture de soi. Corps écrit, n. 5, p. 3-23, fev. 1983.

GASPARINI, Philippe. Est-il je? - roman autobiographique et autofiction. Paris: Seuil, 2004.

GEFEN. Alexandre. Inventer une vie; réinventer la littérature. Bruxelas: Les Impressions Nouvelles, 2015.

GIDE, André. La porte étroite. In : Romans. Paris: Gallimard, 1958.

GOULET, Alain. La vie d'une femme à des messieurs sans compréhension. Paris: MJW Fédition, 2011.

GUSDORF, Georges. La découverte de soi. Paris: Le Presses Universitaires de France, 1948.

. Conditions et limites de l'autobiographie. Berlim: Duncker \& Humblot, 1956.

. Les écritures du moi. Paris: Odile Jacob, 1991. Lignes de Vie, v. I.

JOUANNY, Sylvie. Autofiction. In: SIMONET-TENANT, Françoise (Org.). Dictionnaire de l'autobiographie: écritures de soi de langue française. Paris: Honoré Champion, 2017, p. 94-97.

LACAN, Jacques. Écrits. Paris: Seuil, 1966.

LECARME, Jacques; LECARME-TABONE, Eliane. L'Autobiographie. Paris: Armand Colin, 1997.

- Origines et évolution de la notion d'autofiction. In: BLANCKEMAN,Bruno; MURA-BRUNEL, Aline; DAMBRE, Marc (Org.). Le roman français au tournant du $\mathbf{X X I}^{\mathrm{e}}$ siècle. Paris: Sorbonne Nouvelle, 2004. p. 13-23.

LEJEUNE, Philippe. Moi aussi. Paris: Seuil, 1986.

. Le pacte autobiographique [1975]. Paris: Seuil, 1996.

. L'autobiographie en France [1971]. Paris: Armand Colin, 1998.

. Signes de vie. Paris: Seuil, 2005.

MONDIANO, Patrick. Un pedigree. Paris: Gallimard, 2005.

MONTÉMONT, Véronique. Les vrais monnayeurs. Magazine Littéraire: L'écriture de Soi, n. 530, p. 55, avr. 2013.

PUECH, Jean-Benoît. Fiction biographique et biographie fictionnelle; l'auteur en représentation (2013). Disponível em: <http://books.openedition.org/enseditions/ docannexe/image/4502/img-2.jpg>. Acesso em: 10 nov. 2017.

RABATÉ, Dominique. Le chaudron fêlé: écarts de la littérature. Paris: José Corti, 2006. 
ROUSSEL, Frédérique. Philippe Vasset: de passage secret. Libération, 22 août 2013. Disponível em: http://next.liberation.fr/livres/2013/08/22/philippe-vasset-de-passage-secret_926385. Acesso em: 10 nov. 2017.

SCHWOB, Marcel. Vies imaginaires. Paris: Charpentier et Fasquelle, 1896.

SIMONET-TENANT, Françoise (Org.). Dictionnaire de l'autobiographie; écritures de soi de langue française. Paris: Honoré Champion, 2017.

VIART, Dominique; VERCIER, Bruno. La littérature française au présent. Paris: Bordas, 2005.

VILAIN, Philippe. Défense de Narcisse. Paris: Grasset, 2005.

. L'autofiction en théorie. Chatou: La Transparence, 2009.

Recebido em: 31/08/2017

Aceito em: 15/12/2017 